\title{
Endophytic microorganisms as potential growth promoters of banana.
}

\begin{abstract}
The potential of endophytic microorganisms in promoting the growth of their host plant was determined by artificially introducing five isolates (bacterial and fungal strains: UPM31F4, UPM31P1, UPM14B1, UPM13B8, UPM39B3) isolated from the roots of wild bananas into both healthy and diseased banana plantlets (Berangan cv. Intan). The response of the host plants to endophytic infection was assessed by measuring the change in four growth parameters: plant height, pseudostem diameter, root mass and total number of leaves. The endophytes tested as growth promoters were found to have a significant effect in both healthy and Fusarium-infected (diseased) plantlets. In both experimental systems, the bacterial isolate UPM39B3 (Serratia) and fungal isolate UPM31P1 (Fusarium oxysporum) showed promising growth-promoting properties. Isolate UPM39B3 (Serratia) induced the largest increases in all four growth parameters in healthy plantlets $-3.14 \mathrm{~cm}$ (height), $1.12 \mathrm{~cm}$ (pseudostem diameter), $2.12 \mathrm{~g}$ (root mass) and 1.12 (total number of leaves plant-1) - followed by isolate UPM31P1 (Fusarium oxysporum). The beneficial effect of UPM39B3 (Serratia) and UPM31P1 (Fusarium oxysporum) was also reflected in the diseased plantlets, where pretreatments with the isolates either singly (T6: UPM31P1; T8: UPM39B3) or in a mixture (T7: UPM31P1 + UPM39B3; T9: UPM14B1 + UPM13B8 + UPM39B3) were able to sustain the growth of plantlets, with significantly higher growth values than those in diseased plantlets that were not infected with endophytes (T10: FocR4). These results demonstrate the economic significance of these endophytic isolates, particularly UPM39B3 (Serratia) and UPM31P1 (Fusarium oxysporum), both as potential growth promoters of banana and as agents rendering tolerance towards Fusarium wilt as a strategy in the management of Fusarium wilt of banana via improved vegetative growth.
\end{abstract}

Keyword: Endophytes; Banana; Fusarium wilt; Growth promoters. 